\title{
Emergência de Infecção por Trichosporon Asahii em Pacientes Portadores de Insuficiência Cardíaca em Unidade de Terapia Intensiva Cardiológica. Relato de Caso e Revisão da Literatura*
}

\author{
Trichosporon Asahii an Emerging Etiologic Agent of Fungal \\ Infection and Colonization in Heart Failure Patients in Intensive \\ Care Unit. Case Report and Literature Review
}

\author{
Antônio Aurélio de Paiva Fagundes Júnior', Ricardo Tavares de Carvalho', \\ Rinaldo Focaccia', Juliana Gabriela Fernandez', Hélia Beatriz Nunes de Araújo', \\ Tânia Mara Varejão Strabelli', Liliane Kopel', Silvia G. Lage ${ }^{1}$
}

\section{RESUMO}

JUSTIFICATIVA E OBJETIVOS: As infecções fúngicas por Trichosporon Asahii têm sido cada vez mais freqüentes nas últimas duas décadas. Quadros graves com alta mortalidade são tradicionalmente descritos em pacientes neutropênicos com câncer. Recentemente, a infecção tem ocorrido também em outros grupos de pacientes. O objetivo deste estudo foi descrever a crescente prevalência de Trichosporon asahii em unidade de terapia intensiva cardiológica (UTIC), com perfil de pacientes habitualmente não susceptíveis a tal infecção fúngica, relatar um caso clínico e revisão da literatura.

RELATO DO CASO: Paciente do sexo feminino, 85 anos, com antecedentes de hipertensão arterial sistêmica, insuficiência cardíaca (fração de ejeção $=30 \%$ ) e embolia pulmonar, admitida na UTI depois de parada cardiorrespiratória em fibrilação ventricular durante

1. Unidade de Terapia Intensiva do Instituto do Coração (InCor) do HC-FMUSP, São Paulo, SP

*Recebido da Unidade de Terapia Intensiva do Hospital das Clínicas da Faculdade de Medicina da Universidade de São Paulo, São Paulo, SP

Apresentado em 07 de novembro de 2007

Aceito para publicação em 18 de dezembro de 2007

Endereço para correspondência:

Dr. Antônio Aurélio Fagundes Junior

Av. Dr. Enéas de Carvalho Aguiar, $44 / 2^{\circ} \mathrm{A}$

05403-000 São Paulo, SP

Fones: (61) 3253-7182 / 8186-0773

E-mail: antonioaurelio.jr@gmail.com

(C)Associação de Medicina Intensiva Brasileira, 2008 consulta de rotina. Evoluiu sem seqüela neurológica. $\mathrm{O}$ ecocardiograma não revelou alterações em relação ao exame anterior. Não houve alteração dos indicadores de necrose miocárdica. A paciente apresentou falha na extubação traqueal e desmame difícil, necessitando ventilação mecânica prolongada mesmo após traqueostomia. Houve complicações por insuficiência renal aguda e infecções recorrentes (respiratória, urinária e sistêmica), com boa resposta ao tratamento com antibióticos de amplo espectro. Após sete meses de internação na UTI, evoluiu com choque séptico, associado à infecção urinária por Trichosporon asahii, com hemoculturas identificadas pelo mesmo fungo. Iniciado tratamento com anfotericina B lipossomal ( $5 \mathrm{mg} / \mathrm{kg} / \mathrm{dia}$ ). Apesar do uso associado de vancomicina e imipenem, houve piora clínica progressiva. Hemoculturas colhidas no sétimo dia de uso de antifúngico revelaram-se negativas, porém a urocultura ainda revelou o crescimento de T. asahii. Evoluiu com óbito após 18 dias de tratamento, por falência de múltiplos órgãos.

CONCLUSÕES: O aumento da gravidade dos pacientes internados nas UTI e o uso disseminado de antibióticos de amplo espectro têm possibilitado o surgimento de infecções por fungos incomuns. As infecções graves por Trichosporon asahii, descritas como restritas a pacientes imunossuprimidos, oncológicos e hematológicos, têm sido freqüentemente encontradas em pacientes idosos, com insuficiência cardíaca grave e com alta mortalidade intra-hospitalar, internados em UTI. Deve-se estar atento à possibilidade da emergência de infecções por fungos não usuais em pacientes com este perfil clínico.

Unitermos: infecção, insuficiência cardíaca, Trichosporon asahii, UTI 


\section{SUMMARY}

BACKGROUND AND OBJECTIVES: Infection with the non-Candida yeast species Trichosporon have been recognized with increasing frequency over the last two decades. Invasive disease due to trichosporonosis has been reported from neutropenic patients with cancer and the mortality is high. Recently, others groups of patients have become susceptible to this rare fungi. We report the emerging of infection with pathogenic Trichosporon asahii in severely ill heart failure patients in a tertiary cardiological intensive care unit (CICU). We describe our data, and report a fatal case of disseminated trichosporonosis in a patient with heart failure. We also review literature pertaining to $T$. asahii infections.

CASE REPORT: An 85 year-old woman with a history of hypertension, heart failure (ejection fraction (EJ): $30 \%$ ) and pulmonary embolism was admitted to a medical cardiological ICU after cardiac arrest (ventricular fibrillation) resuscitated during a routine consultation. There were no neurological sequelae and the echocardiogram revels no changes, neither the cardiac biomarkers. Ventricular fibrillation was considered secondary to heart failure. The patient had extubation failure and difficult weaning needing long term mechanical ventilation even after tracheostomy. Her hospital course was complicated by acute renal failure and recurrent respiratory, urinary and systemic bacterial infections, which responded to broad-spectrum antibiotics. After a temporary improvement she developed urinary infection and subsequent septic shock. Cultures of urine and blood specimens grew $T$. asahii. Treatment with liposome amphotericin B ( $5 \mathrm{mg} / \mathrm{kg} /$ day $)$ was started. Despite receiving vancomycin and imipenem, the clinical condition of the patient deteriorates. Blood taken for culture on the seventh day of amphotericin B therapy were negative but urine specimen still grew $T$. asahii. On the eighteenth day of antifungal therapy, the patient died with multiorgan failure.

CONCLUSIONS: The increasing of severely ill patients, and the use of broad spectrum antibiotics, has predisposed the emerging of invasive infections by rare and new opportunistic fungal pathogens. Severe infection related to $T$. asahii, until recently restricted to neutropenic patients with cancer, has been frequently identified in heart failure patients with advanced age. The mortality is high. These data highlights the importance of considering this group of patients as a risk group for $T$. asahii infection.

Key Words: heart failure, ICU, infection, Trichosporon asahii

\section{INTRODUÇÃO}

As infecções fúngicas não associadas às espécies de Cândidas têm aumentado significativamente nas últimas duas décadas. Dentre estas, as infecções por Trichosporon asahii podem apresentar grande variedade de manifestações clínicas, desde acometimento cutâneo superficial até quadros sépticos graves em pacientes imunodeprimidos. Desde o primeiro relato de caso de Trichosporon disseminado em um paciente com leucemia em 1970, casos esporádicos são relatados todos os anos.

O Trichosporon asahii, anteriormente conhecido como Trichosporon beigelii é tradicionalmente descrito como causador de infecções oportunistas levando ocasionalmente a quadros disseminados em pacientes neutropênicos e imunossuprimidos. Walsh e col. ${ }^{1}$, documentou câncer em 55 dos 67 casos revisados, estando a leucemia ou linfoma presente em $60 \%$ deles.

Mais recentemente ${ }^{2}$, outros fatores de risco também têm se associado à infecção: uso de imunossupressores, transplante de órgãos, síndrome da imunodeficiência adquirida (SIDA) ${ }^{3}$, queimaduras extensas ${ }^{4}$, uso de cateter venoso ${ }^{5}$, corticoterapia e cirurgia cardíaca valvar6.

O objetivo deste estudo foi descrever a crescente prevalência de Trichosporon asahii em unidade de terapia intensiva cardiológica (UTIC), com perfil de pacientes habitualmente não susceptíveis a tal infecção fúngica, relatar um caso clínico e revisão da literatura.

\section{RELATO DO CASO}

Paciente do sexo feminino, 85 anos com antecedentes de embolia pulmonar e miocardiopatia dilatada idiopática (fração de ejeção = 30\%), em uso de anticoagulante oral, apresentou parada cardiorrespiratória em fibrilação ventricular enquanto aguardava consulta ambulatorial. Após reanimação cardiopulmonar, houve retorno à circulação espontânea, havendo recuperação completa do nível de consciência. O ecocardiograma não revelou alterações em relação ao exame anterior. Não houve alteração dos indicadores de necrose miocárdica. A fibrilação ventricular foi considerada secundária à miocardiopatia de base. A paciente foi extubada após $72 \mathrm{~h}$ de ventilação mecânica, porém houve falha, sendo re-intubada no mesmo dia. Evoluiu com difícil desmame da ventilação mecânica e derrames pleurais bilaterais de repetição com múltiplas toracocenteses 
e foi traqueostomizada. Apresentou seis infecções nosocomiais documentadas durante a internação na UTI (dois episódios de pneumonia associada à ventilação mecânica, dois episódios de infecção do trato urinário e dois episódios de infecção de corrente sanguínea). Após sete meses de internação na UTI, evoluiu com infecção do trato urinário sintomática (febre e piúria) causado por Trichosporon asahii seguida de hemocultura positiva para o mesmo fungo. Foi iniciado tratamento com anfotericina lipossomal. Apesar do tratamento, evoluiu com quadro de choque séptico, sendo administrados fármacos vasoativos (noradrenalina e dobutamina), além de ampliado espectro antimicrobiano com vancomicina e imipenem. Manteve-se instável hemodinamicamente por duas semanas, sem êxito na suspensão de noradrenalina. Foram colhidas novas culturas após uma semana de tratamento com antifúngico e os antimicrobianos. A hemocultura revelou-se negativa, porém a urocultura ainda apresentava crescimento de Trichosporon asahii. Após 18 dias de uso de anfotericina lipossomal, vancomicina e imipenem, a paciente evoluiu com choque refratário e óbito.

\section{DISCUSSÃO}

O Trichosporon beigelii é um fungo raro, que pode ser encontrado colonizando humanos mas também pode causar infecções superficiais e profundas. Até recentemente, a maioria dos Trichosporon isolados em humanos eram classificados como T. beigelii ou T. cutaneum. Em 1992, Guero e col. propuseram uma revisão taxonômica dos Trichosporon ${ }^{7}$. Subseqüentemente, Sugita e col. ${ }^{8}$ expandiram a proposta reconhecendo seis espécies implicadas em várias infecções em humanos. Destes, T. asahii e T. mucoides estão envolvidos em infecções profundas enquanto $T$. asteróides, $T$. ovóides e $T$. cutaneum são responsáveis por piedra branca e outras infecções superficiais. A sexta espécie, T. inkin é responsável tanto por infecções superficiais como profundas $^{9}$ e recentemente, Moylett e col. ${ }^{10}$ relataram casos de infecção por $T$. pullulans em pacientes com doenças granulomatosas e revisaram cinco casos adicionais da literatura.

O nome $T$. beigelii, amplamente utilizado na literatura, foi descaracterizado já que não houve descrição adequada ou cultura com identificação precisa dessa espécie. Acredita-se que a maioria dos casos de infecção por Trichosporon atribuídos previamente ao T. beigelii e T. cutaneum foram provavelmente causados por T. asahii ${ }^{11}$.
Os principais locais de infecção da Trichosporonose são os tratos respiratório, gastrintestinal e urinário conhecido como locais freqüentes de colonização e posterior disseminação hematológica.

O caso de Trichosporonose disseminada relatado releva a infecção em pacientes freqüentemente internados em UTI: idosos e com insuficiência cardíaca.

$\mathrm{Na}$ UTI clínica do Instituto do Coração do Hospital das Clínicas da Faculdade de Medicina da Universidade de São Paulo (InCor - HC- FMUSP), constituída por 60 leitos, os dados relativos à infecção por Trichosporon asahii, obtidos através de dados coletados pela comissão de controle de infecção hospitalar $(\mathrm{CClH})$, são os seguintes:

- No período de abril de 2000 até maio de 2005 foram observadas 24 infecções urinárias por $T$. asahii, 71\% em homens, com média de idade de $68 \pm 12$ anos. A fração de ejeção (FE) média medida pelo ecodopplercardiograma foi de $42 \% \pm 19 \%$, sendo menor que $40 \%$ em $62 \%$ dos pacientes. Cinqüenta por cento dos pacientes apresentavam insuficiência renal (definida por creatinina sérica maior que $2 \mathrm{mg} / \mathrm{dL}$ ). Os pacientes apresentaram uma média de 2,6 episódios de infecção nosocomial antes do diagnóstico de Trichosporonose. O tempo médio entre a internação na UTI e o diagnóstico da infecção fúngica foi de 34 dias e a mortalidade hospitalar de $83 \%$.

- Em outro grupo, neste mesmo período, foi detectada colonização do trato urinário por $T$. asahii em 20 pacientes; $70 \%$ homens, com média de idade de $73 \pm 11$ anos, FE média de $40 \% \pm 14 \%, 65 \%$ com FE menor que $40 \%$; $55 \%$ desse grupo apresentaram insuficiência renal. A mortalidade hospitalar foi $85 \%$

Desta forma, pôde-se identificar em nosso serviço que o perfil de pacientes com infecções por $T$. asahii constituiu-se por pacientes com idade aproximada de 70 anos, com disfunção ventricular esquerda grave; e, em grande parte com insuficiência renal associada, além de internação prolongada e infecções de repetição. Tais pacientes, em número cada vez mais freqüente nas UTI, são susceptíveis às infecções descritas somente em pacientes gravemente imunossuprimidos. Assim, talvez a infecção por $T$. asahii represente um indicador de extrema gravidade neste grupo de pacientes, o que pode ser verificado pela mortalidade hospitalar elevada, encontrado tanto no grupo com infecção como na colonização do trato urinário por este fungo.

O diagnóstico de infecção por $T$. asahii deve ser feito a partir de culturas de urina, escarro, lavado broncoalveo$\operatorname{lar}^{12}$, líquido peritoneal, líquor e de tecidos. Biópsias e 
culturas de pele em regiões acometidas podem ser úteis para auxiliar o diagnóstico e podem revelar eventos trombóticos por vasculite causada pelo fungo ${ }^{13}$. Hemoculturas são geralmente positivas na infecção disseminada.

O tratamento da fungemia por Trichosporon é difícil e a mortalidade é alta, variando de $35 \%$ até $80 \%{ }^{14}$. Além disso, os métodos para determinação da susceptibilidade in vitro dos vários antifúngicos não estão padronizados e as concentrações inibitórias mínimas (MIC) para o Trichosporon ainda não foram estabelecidas.

Até o momento, não há tratamento de escolha e poucos dados estão disponíveis em relação à susceptibilidade do $T$. asahii aos agentes antifúngicos. O fungo é conhecido por apresentar susceptibilidade variável à anfotericina $\mathrm{B}$ (MIC 0,01 a $4 \mu \mathrm{g} / \mathrm{mL}$ ) e estudos laboratoriais revelam a relativa resistência a este agente ${ }^{10,13}$. É relatada tanto sensibilidade como resistência à flucitocina. Os triazólicos (miconazol, itraconazol, cetoconazol e voriconazol) apresentam maior atividade in vitro em relação a anfotericina $B$ e alguns autores recomendam tratamento combinado com esse último ${ }^{15}$.

À luz dos conhecimentos atuais e dos dados in vitro, a anfotericina $B$ não pode ser considerada terapia de primeira linha para o tratamento da Trichosporonose invasiva. Além disso, evidências atuais sugerem não haver espaço para o uso da caspofungina ${ }^{16}$. Os triazólicos, isolados ou em combinação, tornaram-se os fármacos de escolha. Dentre estas, o voriconazol parece apresentar um bom perfil ${ }^{17}$.

\section{REFERÊNCIAS}

01. Walsh TJ, Newman KR, Moody M et al - Trichosporonosis in patients with neoplastic disease. Medicine, 1986;65:268-279.

02. Chowdhary A, Ahmad S, Khan ZU et al - Trichosporon asahii as an emerging etiologic agent of disseminated trichosporonosis: A case report and an update. Indian J Med Microbiol, 2004;22:16-22.

03. Barchiesi F, Morbiducci V, Ancarani F et al - Trichosporon beigelii fungaemia in an AIDS patient. AIDS, 1993;7:139-140.

04. Hajjeh RA, Blumberg HM - Bloodstream infection due to Trichosporon beigelii in a burn patient: case report and review of therapy. Clin Infect Dis, 1995;20:913-916.

05. Kontoyiannis DP, Torres HA, Chagua M et al - Trichosporonosis in a tertiary care cancer center: risk factors, changing spectrum and determinants of outcome. Scand J Infect Dis, 2004;36: 564-569.

06. Martinez-Lacasa J, Mana J, Niubo R et al - Long-term survival of a patient with prosthetic valve endocarditis due to Trichosporon beigelii. Eur J Clin Microbiol Infect Dis, 1991;10:756-758.

07. Gueho E, Smith MT, de Hoog GS et al - Contributions to a revision of the genus Trichosporon. Antonie van Leeuwenhoek, 1992;61:289-316.

08. Sugita T, Nishikawa A, Ikeda R et al - Identification of medically relevant Trichosporon species based on sequences of internal transcribed spacer regions and construction of a database for Trichosporon identification. $J$ Clin Microbiol, 1999;37:1985-1993.

09. Mussa AY, Singh VK, Randhawa HS et al - Disseminated fatal trichosporonosis: First case due to Trichosporon inkin. J Mycol Med, 1998;8:196-199.

10. Moylett EH, Chinen J, Shearer WT - Trichosporon pullulans infection in 2 patients with chronic granulomatous disease: an emerging pathogen and review of the literature. J Allergy Clin Immunol, 2003;6:1370-1374.

11. Gueho E, Improvisi L, de Hoog GS et al - Trichosporon on humans: a practical account. Mycoses, 1994;37:3-10.

12. Tashiro $\mathrm{T}$, Nagai $\mathrm{H}$, Nagaoka $\mathrm{H}$ et al - Trichosporon beigelii pneumonia in patients with hematologic malignancies. Chest, 1995;108:190-195.

13. Nahass GT, Rosenberg SP, Leonardi CL et al - Disseminated infection with Trichosporon beigelii. Report of a case and review of the cutaneous and histologic manifestations. Arch Dermatol, 1993;129:1020-1023.

14. Walsh TJ, Melcher GP, Rinaldi MG et al - Trichosporon beigelii: an emerging pathogen resistant to amphotericin B. J Clin Microbiol, 1990;28:1616-1622.

15. Wolf DG, Falk R, Hacham M et al - Multidrug-resistant Trichosporon asahii infection of nongranulocytopnenic patients in three intensive care units. $J$ Clin Microbiol, 2001;39:4420-4425.

16. Espinel-Ingroff A - Comparison of In vitro activities of the new triazole SCH56592 and the echinocandinas MK-0991 (L-743,872) and LY303366 against opportunistic filamentous and dimorphic fungi and yeasts. $\mathrm{J}$ Clin Microbiol, 1998;36:2950-2956.

17. Falk R, Wolf DG, Shapiro $M$ et al - Multidrug-resistant Trichosporon

asahii isolates are susceptible to voriconazole. J Clin Microbiol, 2003;41:911. 